\title{
Évolution du ruissellement et du volume d'eau ruisselé en surface urbaine. Étude de cas : Bordeaux 1984-2014, France
}

\author{
Ionel HAIDU ${ }^{1}$, Kinga IVAN ${ }^{2}$ \\ 1. Université de Lorraine, Laboratoire LOTERR - EA 7304, 57045 Metz, France, ionel.haidu@univ-lorraine.fr \\ 2. Babeş-Bolyai University of Cluj-Napoca, 400006 Cluj-Napoca, Romania,ivan_kinga@yahoo.com
}

\begin{abstract}
RÉSUMÉ. - Cette étude a visé l'identification des tendances de modification des cheminements naturels des eaux et du volume d'eau ruisselé en surface urbaine. L'identification des réseaux d'écoulement en surface couverte, respectivement non couverte, sur la base des Modèle Numérique de Terrain - MNT et des Modèle Numérique de Surface - MNS, a mis en évidence la manière dont le cheminement naturel de l'eau a été modifié par les obstacles existants à la surface du terrain. Les Surfaces Totales Imperméables - STI pour les années 1984, 1991, 2002 et 2014 ont été extraites sur la base des images Landsat. La tendance du ruissellement de l'eau pour la période 1984-2014, pour un sous-bassin versant de Bordeaux, a été démontrée à travers la modélisation hydrologique HEC-HSM sur la base des pourcentages des Surfaces Effectives Imperméables - SEI calculés. Les résultats ont mis en évidence la modification des cheminements naturels dans l'aire étudiée et une tendance à l'augmentation du volume d'eau ruisselé. Ces tendances sont le résultat de l'urbanisation et de l'industrialisation et requièrent la mise en place de mesures pour leur atténuation à l'avenir.
\end{abstract}

Mots-clés : hydrologie urbaine, Landsat, modèle numérique de surface, surfaces effectives imperméables, crue urbaine

\section{Urban runoff pathways and surface water volumes evolution. Case study: Bordeaux 1984-2014, France}

\begin{abstract}
This study aimed to identify trends change in natural pathways of urban runoff and of the water volumes into a highly urbanized area of city Bordeaux, France. The identification of the city's flow paths on urbanized terrain and natural land surface based on Digital Terrain Model and Digital Surface Model, highlighted how the natural pathways of the runoff were been modified by successive buildings and other barriers of the urbanized ground surface. On the base of Landsat images, the Total Impervious Area for 1984, 1991, 2002 and 2014 were extracted and the Effective Impervious Areas were calculated. The tendency of the runoff for the period 1984-2014, for a sub-watershed of Bordeaux, was demonstrated through the HEC-HSM hydrologic modeling. The results highlighted the modification of natural paths in the studied area, and a tendency to increase the volume of water runoff. These trends are the result of urbanization and industrialization and require the implementation of mitigation measures in the future.
\end{abstract}

Key-words: Urban hydrology, Landsat, Digital Surface Model, Effective Impervious Areas, Urban flood

\section{INTRODUCTION}

En milieu urbain, le ruissellement de l'eau est influencé par la nature des surfaces, la pente du terrain et l'intensité des précipitations. Sur les surfaces perméables, une partie de l'eau s'infiltre, tandis que sur les surfaces imperméables, l'eau a tendance à s'écouler le long des rues et des trottoirs, en suivant des voies préférentielles de transport de l'eau en fonction de la pente du terrain. Cette étude a visé la mise en évidence de ces voies préférentielles de transport de l'eau et la réponse hydrologique à la variabilité des Surfaces Effectives Imperméables (SEI), à travers l'analyse d'une aire de moins de $1 \mathrm{~km}^{2}$ de la ville de Bordeaux (France). Les surfaces imperméables d'un bassin versant urbain sont réparties en deux catégories : les Surfaces Imperméables Directement Connectées au système de drainage urbain et les Surfaces Indirectement Connectées au système de drainage urbain. Les Surfaces Directement Connectées au système de drainage urbain sont les Surfaces Effectives Imperméables (SEI) et celles-ci contribuent directement au volume d'eau écoulée à la surface d'un bassin versant urbain. Les Surfaces Directement Connectées et Indirectement Connectées au système de drainage urbain forment les Surfaces Totales Imperméables (STI). Pour cette étude, les STI et les SEI ont été estimées sur la base d'images satellitaires. Elles représentent la base pour l'évaluation de la réponse hydrologique à la variabilité de ces surfaces entre 1984 et 2014 .

La ville de Bordeaux est située dans le sud-ouest de la France, dans le département de la Gironde, ayant une altitude comprise entre 1 et $42 \mathrm{~m}$. Elle présente une topographie plane et une localisation géographique qui lui offre de larges possibilités d'extension urbaine. La ville a un climat océanique et des précipitations fréquentes, bien réparties tout au long de l'année. Selon les données statistiques de 2011, la population de la ville de Bordeaux était de 239399 habitants [Insee, 2014]. De la surface totale de $49,36 \mathrm{~km}^{2}$ de la ville de Bordeaux, pour la présente étude, une aire de moins de $1 \mathrm{~km}^{2}$ a été prise en compte, située dans la partie ouest de la ville où des modifications significatives du mode d'occupation du sol ont été enregistrées entre 1984 et 2014. Briat 
et Bourgogne [1992] ont remarqué pour la Communauté Urbaine de Bordeaux l'imperméabilisation croissante des bassins versants et des réseaux qui n'ont pas été conçus initialement pour une urbanisation aussi développée. Donc, ils ont noté que le problème majeur de l'assainissement pluvial est celui des risques d'inondation liés aux orages violents en période estivale et parfois aux fortes hauteurs de précipitations hivernales, ainsi qu'à l'ensemble des ruisseaux qui convergent presque tous vers la partie centrale souvent la plus urbanisée.

$\mathrm{Vu}$ que la nature des surfaces joue un rôle important dans le ruissellement, les Surfaces Totales Imperméables (STI) ont été estimées sur la base des images satellitaires, ainsi que des Surfaces Effectives Imperméables (SEI) pour l'aire urbaine analysée. Les données obtenues par télédétection ont été utilisées dans de nombreuses études en vue de la détection de la variation du type d'occupation du sol en milieu urbain [Deng et al., 2012 ; Shahtahmassebi et al., 2012 ; Yuan and Bauer, 2007] et de l'extraction des surfaces imperméables [Dougherty et al., 2004 ; Falcone and Gomez, 2005 ; Liu et al., 2013 ; Lu and Weng, 2006 ; Lu and Weng, 2009 ; Parece and Campbell, 2013 ; Weng, 2012 ; Xu, 2007 ; $\mathrm{Xu}, 2010$; Yuan et al., 2008 ; Zhou and Wang, 2008]. La connaissance des Surfaces Effectives Imperméables est très importante car celles-ci contribuent directement au ruissellement [Pomeroy et al., 2013]. Pour mettre en évidence la manière dont la nature des surfaces influe sur le ruissellement, le volume d'eau ruisselé dans l'aire d'étude a été déterminé en tenant compte des valeurs des SEI pour les années 1984, 1991, 2002 et 2014. La connaissance de la tendance du volume d'eau ruisselé et du cheminement superficiel en milieu urbain est très importante pour la délimitation des zones exposées aux inondations urbaines.

\section{BASE DE DONNÉES}

Dans l'analyse de la tendance du ruissellement de l'eau en surface urbaine, pour la représentation numérique du terrain, un Modèle Numérique de Terrain - MNT et un Modèle Numérique de Surface - MNS ont été utilisés, car ils sont censés contenir certaines caractéristiques existantes à la surface du terrain : bâtiments, arbres, parkings etc. [Nielsen et al., 2008].

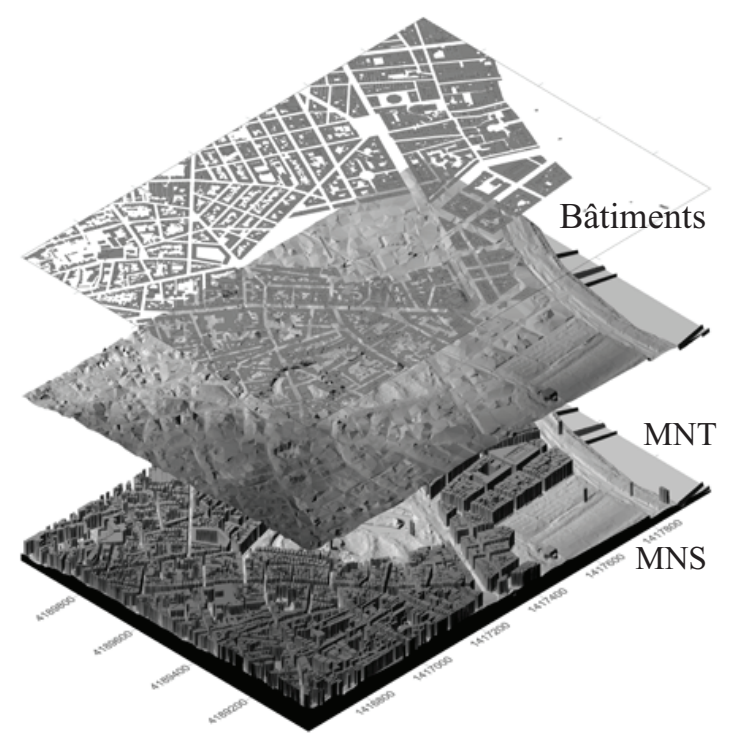

Figure 1 : Schéma du Modèle Numérique de Surface.
Le Modèle Numérique de Terrain pour la ville de Bordeaux, obtenu à partir de la base de données LACUB [LACUB, 2014], a été réalisé à l'aide de la technologie LIDAR, en juillet-août 2012. Ce MNT a permis l'analyse des voies préférentielles d'écoulement de l'eau en surface urbaine non couverte, donc sans bâtiments, végétation, ponts etc. Pour une représentation plus proche de la réalité, un Modèle Numérique de Surface a été réalisé. Celui-ci comprend certaines caractéristiques de la surface du terrain représentant de vrais obstacles pour l'eau : bâtiments, arbres, clôtures en béton, ponts etc.

De toutes ces caractéristiques, le rôle le plus important dans le ruissellement de l'eau revient aux bâtiments, raison pour laquelle ceux-ci ont été inclus dans le MNS. Le bâti de la ville de Bordeaux a été extrait des Plans Locaux d'Urbanisme aux 1/5000 obtenus de la même base de données LACUB. A partir du MNT et des bâtiments, le MNS a été réalisé dans le but d'évaluer la tendance de l'eau lorsqu'elle s'écoule en surface de terrain couvert de bâtiments (Fig.1).

La croissance des surfaces occupées par les bâtiments a déterminé l'extension des surfaces imperméables de l'aire urbaine analysée. Des images satellitaires Landsat ont été utilisées pour l'évaluation de la tendance du volume d'eau ruisselé en surface du terrain suite à l'extension des surfaces imperméables. Les Surfaces Totales Imperméables et perméables de la ville de Bordeaux ont été extraites, pour les quatre années de référence, à partir de quatre scènes Landsat captées les 12 septembre 1984, 22 juillet 1991, 26 juin 2002 et 7 mars 2014, toutes à résolution spatiale de $30 \mathrm{~m}$. Les cartes thématiques ainsi obtenues ont été utilisées dans l'évaluation de l'impact de l'extension des Surfaces Effectives Imperméables sur le régime de l'eau écoulée à la surface du terrain, entre 1984 et 2014. Les effets de l'extension des Surfaces Effectives Imperméables sur le volume d'eau écoulé entre 1984 et 2014 ont été estimés sur la base des précipitations horaires enregistrées les 26-27 juillet 2013. Les données horaires de précipitations enregistrées à la station météorologique de Bordeaux-Mérignac ont été obtenues de l'archive INFOCLIMAT France [Infoclimat, 2014].

\section{MÉTHODOLOGIE}

\section{III.1. Identification des voies préférentielles d'écoulement de l'eau à la surface du terrain}

L'identification des voies préférentielles de transport de l'eau a été réalisée sur la base de la technique rolling ball, au moyen de l'outil Arc Hydro disponible sous ArcGIS 10.x. En utilisant les données numériques de relief, sur la base des directions d'écoulement et d'accumulation de l'eau, les voies d'écoulement de l'eau ont été identifiées pour une zone de drainage de 2,5 ha de l'aire d'étude choisie. La méthodologie de l'identification des voies préférentielles d'écoulement sur une zone sèche du relief a supposé de déterminer la direction d'écoulement et d'accumulation de l'eau et de définir les flux.

Les MNT et les MNS ont permis de déterminer les voies d'écoulement en surface de terrain respectivement non couverte et couverte. La représentation des voies d'écoulement en surface non couverte a mené à la mise en évidence des voies que l'eau a suivi avant que la surface du terrain ne soit recouverte de divers obstacles (bâtiments, clôtures en béton, ponts). Vu que le flux d'eau en surface urbaine est influencé par ces caractéristiques mineures, l'utilisation de données numériques de relief incluant ces obstacles a été nécessaire 
en vue de l'identification des voies que l'eau suit en surface couverte de terrain. De ces caractéristiques, le rôle le plus important dans la direction de l'eau en surface de terrain revient aux bâtiments, raison pour laquelle c'est le seul élément pris en compte dans la réalisation des MNS. La mise en évidence des voies d'écoulement en surface couverte de bâtiments a permis l'identification de la tendance actuelle de ruissellement de l'eau en milieu urbain et la modalité dont les bâtiments ont influencé le changement des voies naturelles d'écoulement de l'eau à la surface.

\section{III.2. Estimation des Surfaces Totales et Effectives Imperméables}

Dans le cadre d'un bassin versant urbain, l'aire occupée par les surfaces imperméables est très importante pour le ruissellement. Les STI et les SEI pour les quatre années de référence ont été extraites des images satellitaires calibrées en valeur de réflectance de la surface, en utilisant la méthode de classification supervisée par maximum de vraisemblance utilisée, entre autres, par Hodgson et al. [2003] ; Parece et Campbell [2013]; Weng [2001]; Xu [2007] pour l'extraction des surfaces urbaines imperméables.

Les Surfaces Effectives Imperméables ont été estimées à l'aide de l'équation Sutherland [Sutherland, 1995] sur la base des valeurs des STI antérieurement obtenues. Les valeurs SEI ont été estimées à l'aide de l'équation de Sutherland (1) pour un sous-bassin versant fortement connecté au réseau de drainage :

$$
\mathrm{SEI}=0.4(\mathrm{STI})^{1.2}
$$

Les Surfaces Effectives Imperméables (SEI) représentent une partie des Surfaces Totales Imperméables (STI) d'un bassin versant directement connecté au réseau de drainage (rues, chemins d'accès, parkings, toitures). La connaissance des valeurs de ces surfaces est très importante pour l'analyse $\mathrm{du}$ processus pluie-ruissellement car il influence directement le volume d'eau ruisselé. Les Surfaces Imperméables d'un bassin versant qui ne sont pas directement connectées au réseau de drainage ne contribuent pas toujours de manière directe à l'écoulement en surface.

\section{III.3. Modélisation hydrologique}

Le modèle Hydrologic Engineering Center-Hydrologic Modeling System (HEC-HMS) a été utilisé en vue de l'identification de la tendance du volume d'eau ruisselé compte tenu des modifications des Surfaces Effectives Imperméables (SEI) de l'aire étudiée, pour la période 1984-2014. Ce modèle a été développé par l'U.S. Army Corps of Engineers (USACE, 2000) et il est employé sur une large échelle dans les simulations du processus pluie-drainage tant dans les bassins versants naturels que dans les petits bassins versants urbains. La modélisation hydrologique permet d'évaluer la réponse hydrologique aux changements survenus dans le type d'occupation du sol pour une période de temps plus longue. L'utilisation du modèle HEC-HMS a conduit à l'estimation de la réponse hydrologique à la variabilité des Surfaces Effectives Imperméables pour la période 1984-2014 sur la base de précipitations horaires. Ce modèle a imposé comme paramètres d'entrée : les valeurs horaires de précipitations, le numéro de courbe d'écoulement (valeur SCS-CN) calculée sur la base de données concernant le type de sol et d'occupation du sol extraites des images satellitaires, tout comme la valeur des Surfaces Effectives Imperméables pour chaque année de référence.

Le modèle hydrologique HEC-HMS utilise des modèles séparés pour représenter les composantes du processus de ruissellement, chacune ayant un nombre variable de méthodes de modélisation (USACE, 2000). De ces modèles, pour la présente étude, seulement le modèle de calcul du volume d'eau ruisselée et celui du ruissèlement direct ont été utilisés. La simulation du volume d'eau ruisselé a été réalisée sur la base de la fonction de production développée par le Soil Conservation Service (SCS-CN) qui relie le cumul de la pluie efficace $P e$ au cumul de la pluie brute $P$, par l'équation :

$$
P_{e}=\frac{\left(P-I_{a}\right)^{2}}{\left(P-I_{a}\right)+S}
$$

où $I_{a}$ désigne les pertes intervenant en début d'événement et $\mathrm{S}$ la capacité de rétention en eau du sol (l'infiltration cumulée, $\mathrm{mm}$ ).

Le modèle exprime l'évolution du coefficient de ruissellement, de 0 lorsque le cumul de pluie brute est inférieur à $I_{a}$, à 1, lorsque le cumul de pluie brute tend vers l'infini. Les paramètres d'ajustement du modèle sont $I_{a}$ et $S$. On admet généralement qu' $I_{a}$ et $S$ sont liés par la relation :

$$
I_{a}=0.2 S
$$

La capacité de rétention en eau $(S, m m)$ peut également être reliée au numéro de la courbe d'écoulement empirique du SCS par la relation :

$$
S=\frac{25400}{C N}-254
$$

La transformation de l'excès de précipitation en écoulement direct a été réalisée sur la base de l'hydrogramme unitaire SCS. Les paramètres d'entrée de modèle du ruissellement direct, selon le modèle de l'hydrogramme unitaire, le temps de retard entre le pic de crue et le pic des précipitations $\left(t_{l a g}\right)$ et la surface du sous-bassin versant ont été déterminés au moyen de HEC-GeoHMS sous ArcGis 10.x. Sur la base du temps de retard $\left(t_{\text {lag }}\right)$, le temps de concentration $\left(T_{c}\right)$ approprié aux sous bassins versants pour les années 1984, 1991, 2002, 2014 (USACE, 2000 ; Gyori et Haidu, 2011) a été calculé :

$$
t_{\text {lag }}=0.6 \times T_{c}
$$

avec $T_{c}$ le temps de concentration de l'hydrogramme de crue. Le temps correspondant au pic de crue $\left(T_{p}\right)$ a été ainsi calculé :

$$
T_{p}=\frac{\Delta t}{2}+t_{\text {lag }}
$$

où $\Delta_{t}$ représente la durée des précipitations en excès et $t_{\text {lag }}$ le temps de retard

La pointe de crue, sommet de l'hydrogramme $\left(U_{p}\right)$ a été ainsi calculé :

$$
U_{p}=2.08 \frac{\mathrm{A}}{T_{p}}
$$

avec $A$ la surface du bassin. 


\section{RÉSULTATS ET DISCUSSIONS}

Les résultats obtenus sur la base des deux modèles numériques, MNT et MNS, ont montré le fait que les voies d'écoulement mises en évidence sur la base des MNS sont beaucoup plus proches de la réalité et qu'elles suivent en général la direction des routes et des trottoirs. On peut observer dans la Fig. 2 que les voies d'écoulement mises en évidence sur la base des MNT qui ne tiennent pas compte de certaines caractéristiques de la surface du terrain (en l'occurrence, les bâtiments) peuvent se superposer aux bâtiments, dans certaines aires. Ces flux représentent les voies suivies par l'eau avant que la surface urbaine ne soit couverte de bâtiments et mettent en évidence un net changement des voies d'écoulement de l'eau en milieu urbain comme résultat des changements du type d'occupation du sol.

Ces différences, obtenues entre les voies d'écoulement de l'eau en surface couverte par rapport à la surface non couverte de terrain, indiquent le fait que, dans les aires urbaines, les bassins versants d'écoulement ne sont plus naturels et qu'ils sont modifiés par les bâtiments, les rues et les caniveaux. Toutes ces caractéristiques de la surface urbaine modifient d'une manière substantielle la direction de l'écoulement naturel de l'eau à la surface du terrain. L'eau des précipitations est forcée, à cause des obstacles existants en surface du terrain urbain (bâtiments), à suivre certaines voies d'écoulement le long des rues, des trottoirs, selon la plus forte pente. Dans les parties les plus basses, l'eau a tendance à s'accumuler dans les petites dépressions où elle peut stationner dans les points bas pour une période plus ou moins longue, en fonction des précipitations tombées et en fonction de la capacité de drainage du réseau de proximité. À cause de l'urbanisation, la modification des voies naturelles d'écoulement de l'eau s'accompagne de l'augmentation du volume d'eau écoulé sur le fond de l'extension des surfaces imperméables. L'urbanisation et l'industrialisation ont conduit à la modification du mode d'occupation du sol au cours du temps. L'urbanisation a déterminé l'augmentation des surfaces imperméables qui contribuent d'une manière significative à l'écoulement de surface, le rôle le plus important revenant aux Surfaces Effectives Imperméables.

Dans cette étude, l'estimation des valeurs des SEI est importante pour la connaissance des surfaces imperméables dans l'aire étudiée qui contribuent directement à l'écoulement de surface et qui influencent le volume d'eau écoulée. Dans l'aire analysée, ces surfaces comprennent les surfaces qui ne permettent pas à l'eau de s'infiltrer dans le sol, tout comme certaines surfaces occupées par les rues, les trottoirs et les toitures. Dans le sous-bassin versant de $0.46 \mathrm{~km}^{2}$ analysé, on a estimé $52,9 \%$ (SEI) et 58,6\% (STI) en 1984 , $72,2 \%$ (SEI) et 76,0\%(STI) en 1991, 82,3\% (SEI) et $84,7 \%$ (STI) en 2002 contribuent directement à l'écoulement de surface.

La différence entre les STI et les SEI, de 5,7\%, 3,2\% et $2.4 \%(1984,1991$ et 2002$)$, dans le sous-bassin versant analysé, représente les surfaces imperméables indirectement connectées au système de drainage urbain, des surfaces qui ne conduisent pas toujours à l'écoulement direct. En 2014 les valeurs des SEI $=$ STI $=97,8 \%$ ce qui met en évidence le fait que, à présent, toutes les surfaces
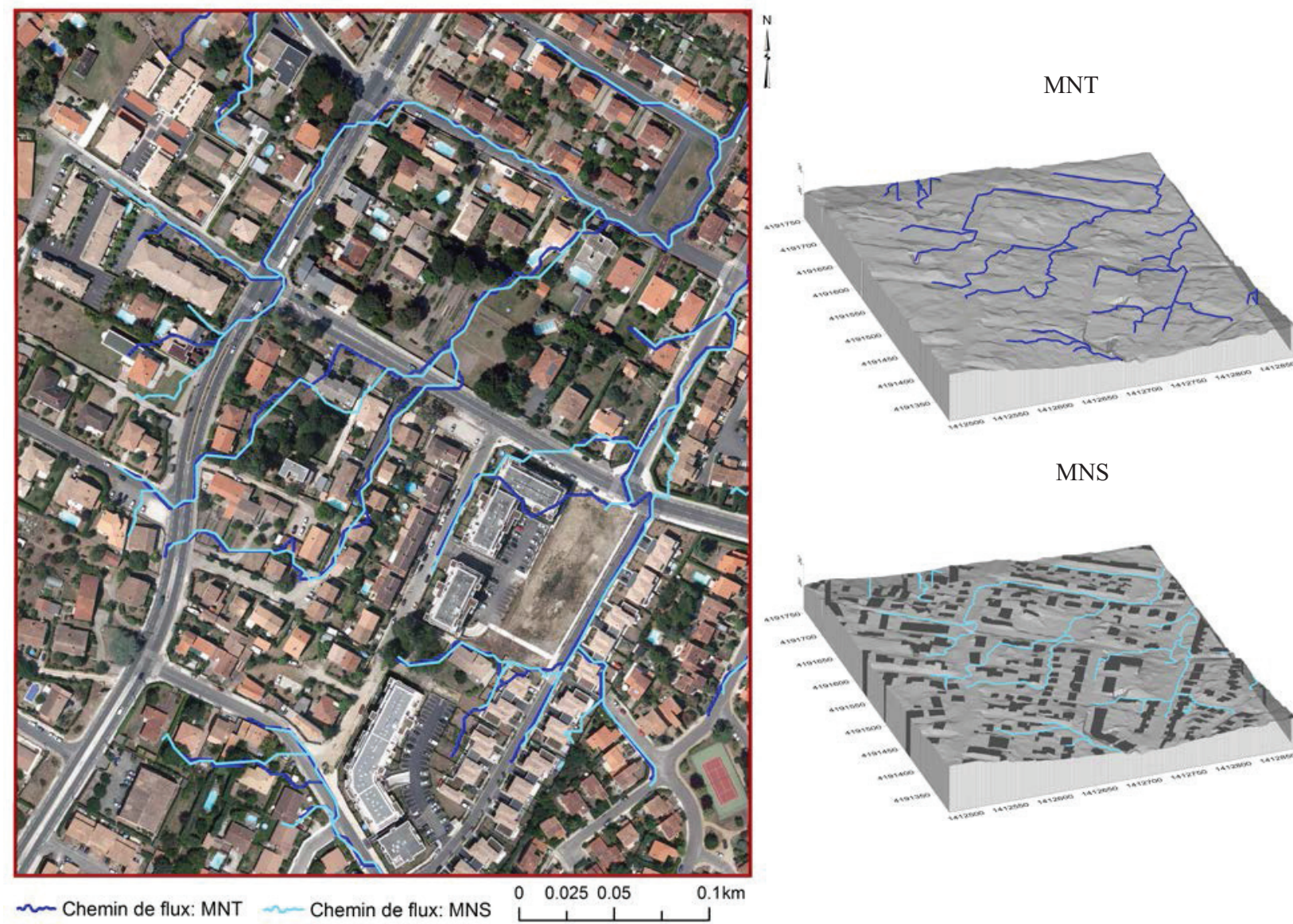

MNS

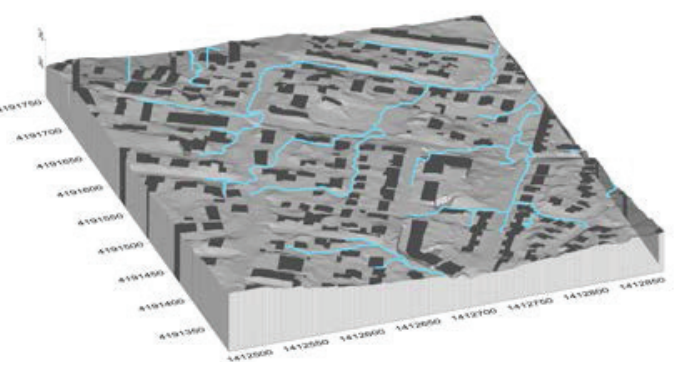

Figure 2 : Voies préférentielles d'écoulement calculées sur la base des MNT et des MNS pour l'aire étudiée. 
imperméables contribuent directement à l'écoulement superficiel de l'eau dans l'aire analysée et cela par rapport aux situations précédentes.

Pour mettre en évidence la modalité dont les changements du type d'occupation du sol ont influencé l'écoulement en milieu urbain entre 1984 et 2014, le volume d'eau écoulée dans le sous- bassin versant analysé a été déterminé pour les valeurs horaires des précipitations tombées les 26 et 27 juillet 2013. Les résultats de la modélisation hydrologique ont montré une tendance à la croissance du volume d'eau écoulé et des pics de crues de 1984 à 2014 et une baisse du total des pertes sur le fond de l'augmentation des valeurs des Surfaces Effectives Imperméables du sous-bassin versant d'écoulement analysé (Fig. 3).

Les séries des hydrogrammes pour chaque année de référence permettent d'observer que, pour la même précipitation, le pic de crue a été beaucoup moins élevé en 1984 qu'en
2014. On assiste à présent à une croissance des flux de crue et du volume d'eau écoulée, dont les conséquences se reflètent directement sur la population affectée de plus en plus par l'eau écoulée en surface de terrain. Suite aux précipitations tombées les 26 et 27 juillet 2013, entre $23 \mathrm{~h} 00$ et $02 \mathrm{~h} 00$, une inondation urbaine a eu lieu à Bordeaux, en provoquant des dégâts immenses, en inondant des garages, des maisons, des sous-sols de maisons, des hôpitaux, tout comme le hall de la gare Saint-Jean et en affectant la circulation sur plusieurs artères à Bordeaux (Fig. 4 et Fig. 5). Toutes les conséquences de la modification des voies d'écoulement de l'eau en surface urbaine et de la tendance de croissance du volume d'eau ruisselé sur le fond de précipitations extrêmes ont à présent des effets dévastateurs. Les conséquences de l'extension du bâti se ressentent de plus en plus en milieu urbain; il s'impose la prise de mesures en vue d'atténuer cette tendance à l'avenir.

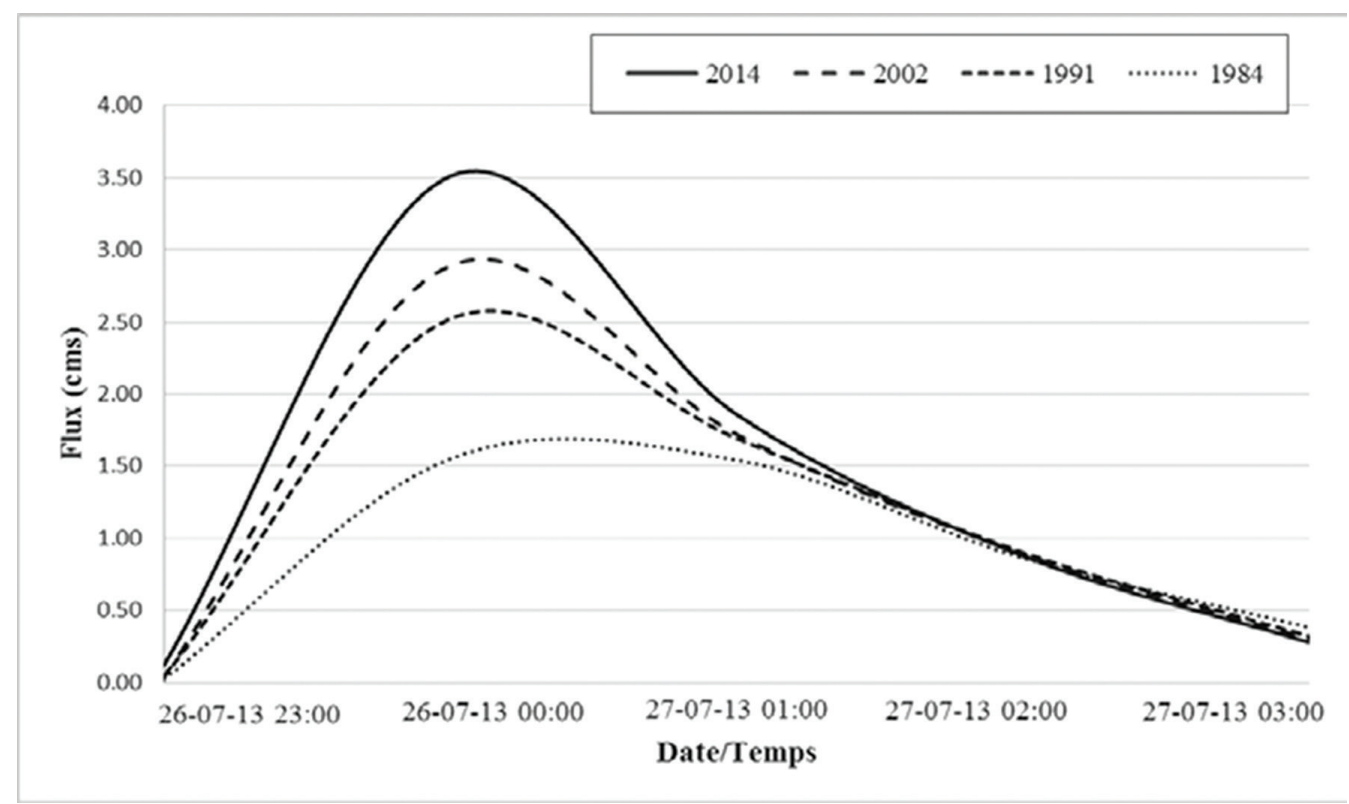

Figure 3 : Hydrogrammes calculées sur la base des valeurs SEI pour la période 1984-2014.

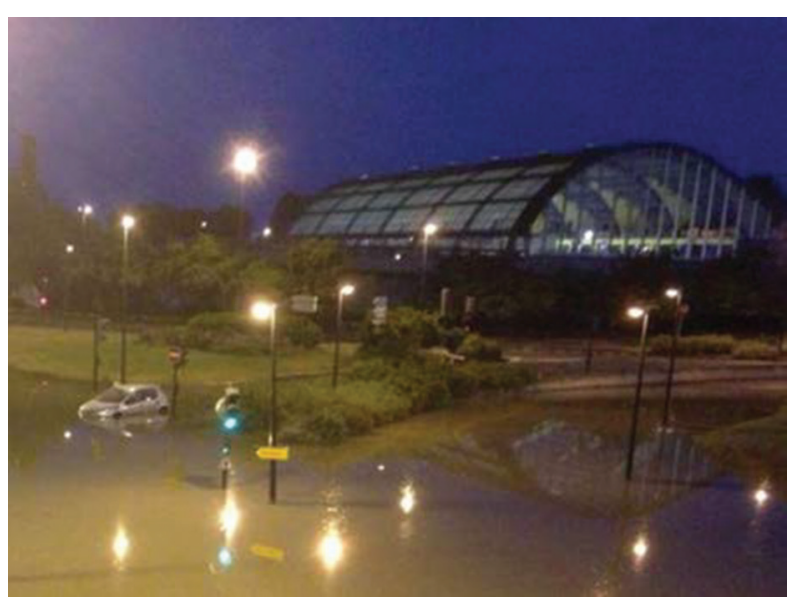

Figure 4 : Rue inondée à Bordeaux. (source: journal « Sud Ouest », 2013).

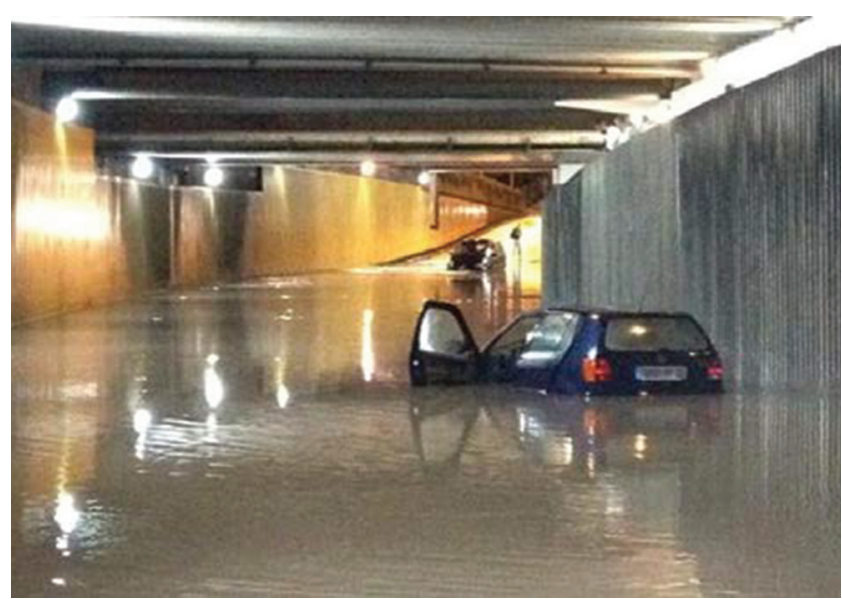

Figure 5 : Passage souterraine inondée à Bordeaux. (source: journal « Sud Ouest», 2013). 


\section{CONCLUSIONS}

Le flux d'eau en surface est directement influencé par les obstacles existants à la surface du terrain, la nature des surfaces, la pente du terrain et l'intensité des précipitations. La délimitation des voies d'écoulement sur la base des MNT et des MNS a permis d'identifier les différences entre les voies d'écoulement de l'eau en surface couverte, par rapport à la surface non couverte du terrain. Ces différences indiquent une nette modification des voies naturelles d'écoulement de l'eau à cause des obstacles existants en surface de terrain, dont les bâtiments ont été analysés. Les obstacles existants à la surface du terrain ont déterminé la modification des voies naturelles d'écoulement, l'eau étant forcée à suivre certaines voies d'écoulement, généralement le long des rues et des trottoirs, ce qui entraîne la constitution de véritables canaux ouverts d'écoulement. Tous ces obstacles existants en surface du terrain sont le résultat de l'urbanisation et de l'industrialisation qui ont conduit à la modification du type d'occupation du sol au fil du temps.

Sur le fond de l'urbanisation et de l'industrialisation, les surfaces imperméables se sont au fur et à mesure développées. De ces surfaces, les Surfaces Effectives Imperméables ont un rôle important, car elles influencent directement le volume d'eau écoulée. Sur la base des Surfaces Totales Imperméables extraites des images satellitaires pour les années 1984, 1991, 2002 et 2014, les Surfaces Effectives Imperméables ont été calculées; la tendance du volume d'eau écoulé entre 1984 et 2014 a été déterminée. Les résultats ont mis en évidence des valeurs de SEI de 52,9\% (1984), 72,2 \% (1991), 82,3\% (2002) et 97,8 \% (2014) qui ont directement contribué à l'écoulement de l'eau en surface dans l'aire analysée. Sur la base des valeurs SEI et suite à la modélisation hydrologique, les résultats en mis en évidence une augmentation du volume d'eau écoulé et des pics de crue entre 1984 et 2014.

Toutes les tendances de modification des voies naturelles d'écoulement de l'eau, du volume d'eau écoulé et des ondes de crue, doivent constituer un signal d'alarme pour les autorités locales en vue de la mise en place de mesures pour atténuer ces tendances à l'avenir.

\section{REFERENCES}

Briat P., Bourgogne P. (1992) - Exemple d'acquisition et de gestion des écoulements sur le réseau Pluvial De La Communauté Urbaine De Bordeaux. La Houille Blanche. 6 437-442

Deng Y., FAn F. \& Chen R. (2012) - Extraction and Analysis of Impervious Surfaces Based on a Spectral Un-Mixing Method Using Pearl River Delta of China Landsat TM/ETM+ Imagery from 1998 to 2008. Sensors. 12 1846-1862

Dougherty M., Dymond R.L., Goetz S.J., Jantz C.A. \& Goulet N. (2004) - Evaluation of impervious surface estimates in a rapidly urbanizing watershed. Photogrammetric Engineering and Remote Sensing. 70 1275-1284

FALCONE J.A., GOMEZ R. (2005) - Mapping impervious surface type and sub-pixel abundance using Hyperion hyperspectral imagery. Geocarto Int. 20 (4) 3-10

GYORI M.M., HAIDU I. (2011) — Unit Hydrograph Generation for Ungauged Subwatersheds. Case Study: the Monoroștia River, Arad County, Romania. Geographia Technica. 6 (2) 23-29

Hodgson M.E., Jensen J.R., Tullis J.A., Riordan K.D. \& ARCHER C.M. (2003) - Synergistic use of LiDAR ad color aerial photography for mapping urban parcel imperviousness. Photogrammetric Engineering and Remote Sensing. 69 973-980
INFOCLIMAT. (2014) - Available from: http://www.infoclimat.fr [Accesed 15 October 2014].

INSEE. (2014) - Institut National de la Statistique et des Etudes Economiques, France. Available from: http://www.insee.fr [Accesed 10 August 2014]

LACUB. (2014) - La Communauté urbaine de Bordeaux. Available from: http://data.lacub.fr/data [Accesed 10 April 2014].

Liu C., Shao Z., Chen M. \& Luo H. (2013) - MNDISI: a multi-source composition index for impervious surface area estimation at the individual city scale. Remote Sensing Letters. 4(8) $803-812$

Lu D., WeNG Q. (2006) - Use of impervious surface in urban landuse classification. Remote Sensing of Environment. 102 146-160

Lu D., WeNG Q. (2009) — Extraction of urban impervious surfaces from IKONOS imagery. International Journal of Remote Sensing. 30(5) 1297-1311

Nielsen N.H., Jensen L.N., Linde J.J. \& Halager P. (2008) Urban Flooding Assessment. 11th International Conference on Urban Drainage, 31st August - 5th September 2008, Edinburgh, Scotland.

Parece T. E., Campbell J.B. (2013) - Comparing Urban Impervious Surface Identification Using Landsat and High Resolution Aerial Photography. Remote Sensing. 5 4942-4960

Pomeroy Lisa J.M., Swan R., Thomson R., Phillips B., Ball J. \& PATHIRAJA S. (2013) - Estimation of imperviousness for a Victorian urban catchment. 8th Victoria Flood Conference, Melbourne, Australia.

Shahtahmassebi A., Zhou L., Wang K., Xu H., Deng J., Li J., Luo R., Wu J. \& Moore N. (2012) - Monitoring rapid urban expansion using a multi-temporal RGB-impervious surface model. Environmental Engineering. 13 (2) 146-158

Sud Ouest. (2013) - Orages : vos photos de Bordeaux sous les eaux. 27 juillet 2013. Available from: http://www sudouest.fr/2013/07/27/le-centre-de-bordeaux-sous-leseaux-1126304-2780.php [Accesed 15 October 2014]

Sutherland R.C. (1995) — Methods for Estimating the Effective Impervious Area of Urban Watersheds. The Practice of Watershed Protection, Article 32. 193-195

U. S. Army Corps Of Engineers (Usace). (2000) - Hydrologic Modeling System HEC HMS - Technical Reference Manual. U. S. Army Corps of Engineers, Hydrologic Engineering Center.

WENG Q. (2001) - Modelling urban growth effects on surface run-off with the Integration of Remote Sensing and GIS. Environ. Manage. 28 737-748

WeNG Q. (2012) - Remote sensing of impervious surfaces in the urban areas: Requirements, methods, and trends. Remote Sensing of Environment. 117 34-49

XU H.Q. (2007) - Extraction of urban built-up land features from Landsat imagery using a thematic-oriented index combination technique. Photogrammetric Engineering and Remote Sensing. 73(12) 1381-1391

Xu H. (2010) - Analysis of impervious surface and its impact on urban heat environment using the normalized difference impervious surface Index (NDISI). Photogrammetric Engineering and Remote Sensing. 76 (5) 557-565

YUAN F., BAUER M.E. (2007) - Comparison of impervious surface area and normalized difference vegetation index as indicators of surface urban heat island effects in Landsat imagery. Remote Sensing of Environment. 106 (3) 375-386

YUAN F., Wu C. \& BAUER M.E. (2008) - Comparison of spectral analysis techniques for impervious surface estimation using Landsat imagery. Photogrammetric Engineering and Remote Sensing. 74(8) 1045-1055

ZHou Y.Y., WANG Y.Q. (2008) - Extraction of impervious, surface areas from high spatial resolution imagery by multiple agent segmentation and classification. Photogrammetric Engineering and Remote Sensing. 74(7) 857-868 